\title{
DIE RICHARD-ANNA-SZENE IN SHAKESPEARES RICHARD III. (I, 2.)
}

"Wenn die könige bau'n, haben die kärrner zu tun." Allein um der werbeszene Richards um lady Anne willen sind viele bedeutende federn in bewegung gesetzt worden. Als kuriosum sei an der spitze erwähnt die ansicht Vershofens, der Anna eine "stolze seele" nennt.") Eine stolze seele! Woraus schliefst er das? Rümelin lehnt die szene überhaupt als ganz unmöglich und künstlerisch verfehlt $a b .{ }^{2}$ ) Andre erklärungen sind vortrefflich, aber nicht vollständig, auch die von Wetz scheint mir nicht ins schwarze zu treffen. Näher kommen Heine und stellenweise Fr. Th. Vischer, am besten ist die erklärung von Mrs. Jameson. Wetz führt ein urteil an ${ }^{3}$ ): es sei eine so schwierige sache, in die geheimsten falten des weiblichen herzens einzudringen, "dafs gerade die erfahrensten psychologen und frauenkenner, wie Stendhal, offen eingestehen, dafs sie immer wieder einzelne erfahrungen machen mulsten, die von ihnen für völlig sicher gehaltenen annahmen widersprachen und ihnen den beweis dafür zu liefern schienen, dafs der mann mit seinen stumpferen organen über manche seiten des weiblichen gemüts überhaupt nur unzulängliches aussagen könne". Vielleicht! Denn Mrs. Jamesons auffassung ist die treffendste. Aber Shakespeare selber! Die kenntnis der weiblichen wie der männlichen seele ist eine sache der

1) In seinem vortrefflichen buch: Charakterisierung durch Mithandelnde bei Shakespeare 8. 83. Bonner Beitr. z. Anglistik XX.

2) G. v. Rumelin, Shakespeare-Studien eines Realisten, s. 106. Stuttgart 1866.

3) Wetz, Die Menschen in Sh.'s Dramen s. 118.

Anglia. N. F. XXIX. 
besonderen psychologischen oder noch besser der rein künstlerischen, divinatorischen begabung. "Es ist daher auch ein meist befolgtes gebot der klugheit", fährt Wetz fort, "wenn der psycholog sich darauf beschränkt, die komplizierteren phänomene des weiblichen seelenlebens, welche ihm aufstofsen, zu erklären, statt apodiktische sätze über die möglichkeit oder unmöglichkeit dieser oder jener handlungsweise bei dem weibe aufzustellen." Es sei darum genaue sachkenntnis auf dem gebiet der weiblichen herzenskunde erforderlich.

Gewils. Es ist aber ganz verfehlt, die weibliche psyche immer als ein fremdes, unheimliches wesen zu betrachten, das sich plötzlich in einen werwolf verwandeln könne. In der männlichen seele sind auch abgründe, woran scheiterte sonst immer die Hamletpsychologie? Mann und weib folgen bestimmten psychologischen gesetzen, die für die gattung mensch gelten, und auch die weibliche seelentätigkeit hat keine sondergesetze, die bisher noch unbekannt geblieben wären. Hier in Lady Anne haben wir überdies nicht einmal ein solches "kompliziertes phänomen"; im gegenteil, dutzendware. "Lady Anne is just one of those women whom we see walking in crowds through the drawing-rooms of the world. The puppets of habit, the fools of fortune, without any particular inclination for vice or any steady principle for virtue; whose actions are inspired by vanity not affection, and regulated by opinion not by conscience: who are good while there is no temptation to be otherwise and ready victims of the first soliciting to evil. .... Cressida is another modification of vanity, weakness and falsehood drawn in stronger colours. The world contains many Lady Annes and Cressidas, polished and refined externally whom chance and vanity keep right, whom chance and vanity lead wrong just as it may happen. When we read in history of the enormities of certain women, perfect scarecrows and ogresses, we can safely hug ourselves in our secure virtue and thank God that we are not as others are: but the wicked women inShakespeare are portrayed with such perfect consistency and truth that they leave us no such resource; they frighten us into reflection, they make us believe and tremble." 1)

1) Mrs. Jameson, Characteristics of Women, Moral, Poetical and Historical. 2. anfl. London 1870. Introduction p. 21. 
Welche schwierigkeit will man eigentlich in dieser szene finden? Dals Anna sich so schnell umstimmen lälst, und dals ihre umstimmung nicht genügend begründet ist. Aber wer hätte dergleichen noch nicht erlebt? Nie an einer edlen frau, - aber an einer unedlen. Fr. Th. Vischer ${ }^{1}$ ) beantwortet seine frage: "Wie sollen wir nun diese Anna beurteilen, als ein sehr geringes wesen?" mit: "Doch wohl nicht." Er nennt sie "ein unedles, wenn auch nicht grobsinnliches weib".2) Vershofen aber bezeichnet sie als eine "stolze seele"; und es ist doch wohl keine wortverdreherei, wenn das mit dem attribut "edel" gleichgesetzt wird. Wie sollen wir beweisen, daIs sie unedel ist? "Auch sie wird zur Chriemhilde, als sie ihrem Hagen gegenübersteht, aber aus ihrem schmerz und ihrem rachedurst redet in klaren, scharfgefügten worten die wahrheit." 3) Trotz dieser äufseren ähnlichkeit der situation aber ist sie keine Kriemhild, die Nibelungenfürstin steht hoch über ihr. Nun lassen sich wohl für alle psychologischen auslegungen beweise bringen, im gebiete der psychologie ist ja der subjektivität der weiteste spielraum gelassen, daher die nicht endenwollende unklarheit auch über diese gestalt. Gäbe es also kein mittel, um einen charakter auf seine grundstruktur zurückzuführen? Doch, seine handlungen. Es scheint fast, als ob dieses erkenntnismittel bei der beurteilung dramatischer persönlichkeiten immer als zu selbstverständlich verachtet würde. In grolsartig furchtbarer folgerichtigkeit nährt Kriemhild ihren wilden rachedurst bis $\mathrm{zu}$ seiner erfüllung. Ihr dämonischer hals bleibt unverändert bis zum ende. Kriembild liâst sich nicht betören, durch nichts, neben ihrer toten liebe ist für keine süfsigkeit des lebens mehr raum. Annas klagerede (II, v. 1-32) ist sehr gewissenhaft und geschieht nach allen regeln der kunst. Bei den alten Juden gab es ja sogar klageweiber von beruf. Pflichtgemäls erhebt sie ihre klage jedesmal von neuem, wenn die träger die bahre hinsetzen, was sollten soust die leute von ihr denken! Ihr jammergeschrei entstammt, ganz klassizistisch, verstandesgemäLser überlegung, nicht wahrem schmerz. Diesen spielt sie nur, weil es

1) Richard III, 8. $270 \mathrm{f}$.

2) Zitiert nach Wetz, s. $118 \mathrm{ff}$.

3) Vershofeu, s. 49. 
sich so schickt für eine trauernde witwe und schwiegertochter, und in dankbarer anerkennung der vorzüge der toten. Sie gibt sich mühe, darum lassen uns ihre klagen kalt. Man lese daraufhin noch einmal I, 2, v. 1-32 durch. Sie bricht nicht in neuen jammer aus über die frischblutenden wunden des toten, sondern macht die herren des gefolges darauf aufmerksam (1l. 55, 56). Wahrer schmerz spricht anders, so in Margarete v. Anjou, so in königin Elisabeth. Zur schau getragener schmerz ist wie ein auf einmal ausgegossenes fafs, wahrer schmerz wie eine unversiegliche quelle; jener hat die stärke des ausbruchs, dieser die beständigkeit vor dem andern voraus. Aber der affekt, sei der wille noch so gut, erschöpft sich bald. Trotz aller aufgewandten anstrengung, gerade wegen dieser, bringt sie nur ein kleinliches keifen heraus. In welcher ihrer reden wollten wir wirklich ergreifenden schmerz erkennen können? Sie steigert sich in eine künstliche wut hinein, die, je schwächer das gespielte gefühl, desto aufserlicher sich kundgibt in schimpfworten und sogar im anspeien, und schliefslich in blols spitzfindigen worten endet (11. 33-146). "It is a quarrel just and reasonable To be revenged on him that slew my husband." An welcher stelle fänden wir wirkliche emporrung, den echten schmerzensschrei einer zu tode getroffenen? Wie armselig gemacht ist das alles gegen Margaretens grofsartige flüche und königin Elisabeths bitterlichen jammer (IV, 9-14), oder gegen Ophelias sanfte herzenstöne (Hamlet III, 1, 158-169). Sie haben wirklich verloren, Anna macht nur andere darauf aufmerksam, gibt eine nachahmung wirklichen schmerzes wie eine geschickte schauspielerin. Kein wunder, wenn sie ihre rolle so schnell ausgespielt hat und sich kirren läfst. Es kommt nicht darauf an, ob das in wenigen augenblicken auf der bühne geschieht oder in einigen jahren. Die möglichkeit überhaupt beleuchtet den charakter. Aufserdem ist von verschiedenen Shakespeare-erklärern 1) hingewiesen worden auf die ideale, nicht wirkliche zeit der bühne, durch die alle vorgänge eine verkürzung erfahren. Auch Hebbels Ernst-Albrecht-szene 2) wird als glaubhaft empfunden; und im grunde liegt ja auch

1) Z. b. O. Ludwig, Shakespeare-Studien. Werke, ansgabe Hesse, s. 54 .

2) Agnes Bernaner $\nabla, 10$. 
das entscheidende moment einer umstimmung in einem angenblick, nur die folgen werden gewöhnlich langsamer erkennbar.

Wenn man Annas umstimmung als nicht genügend begründet betrachtet, so vergifst man vor allen dingen immer, dals ja ihr weiteres verhalten selbst aufschlufs über ihren charakter gibt. "Sie wird nachher 'gute tante Anna' genannt und als arme dulderin beklagt. Anna braucht nur schwach, nur eitel, braucht nicht gemein und schlecht zu sein, um darin von uns verstanden zu werden, dals sie dann sogar in diesem augenblick, vor der leiche ihres schwiegervaters und von seinem und seines sohnes mörder sich rühren läIst." ') Gewils nicht; man kann sich wohl vorstellen, dafs Anna keiner kreatur ein leid tut, dals sie für die kümmernisse ihrer mitmenschen ein bereites mitgefühl hat, ebenso für ihre "neffen"; sie ist eben die "gute tante Anna", die "freundliche schwester". Die kleinbürgerliche Mrs. Tulliver in "The Mill on the Floss" ist auch eine gute fran, aber diese art "güte" entstammt nicht sittlicher gröfse, sondern einer triebhaften gutmütigkeit, wie sie oft die genossin der beschränktheit ist. Wie kann man aber mit Wetz aus den ausdrücken "kind aunt of Gloster", etc. ${ }^{2}$ ) und Annas klage über ihre eigne himverbrannte verblendung ${ }^{3)}$ eine "ungewöhnliche, bis zur schwäche gehende herzensgüte" herauslesen, die "ihr hervorragendster charakterzug" sein soll?!" ") Wohl "ist keine andentung, dals die Richard nicht wohl gesinnten personen Anna wegen ihrer schwäche gering schätzen. Alle beweisen ihr nur aufrichtiges mitleid";s) sie wären ihrem charakter nicht getreu, wenn sie schadenfreude empfänden; die alte herzogin von Gloster und königin Elisabeth sind wirklich gut, anch ohne das machte sie ihr eigenes herzeleid mitftihlend für fremdes; und es mülste schon ein recht roher mensch sein, der noch schadenfreude empfände, wenn er jemand, dem er gleichgültig oder nicht wohlgesinnt war, rettungslos ins sichere verderben treiben sieht. "Nur eines hat sie nicht, charakter. wo kein charakter ist, sind die instinkte des geschlechtes um

1) Vischer, s. $170 \mathrm{f}$.

$\Rightarrow$ IV, $1,1-26$.

3) IV, 1, 54-79.
๑) $S .118 \mathrm{f}$.

5) A. 2. 0. 
so stärker und machen jeden momentansten umschlag möglich." 1) Damit kommen wir an die wurzel.

Die passendste parallele zu Anna geben die MartheMephisto-szenen im Faust: die verliebtheit ihrer natur wird nur noch durch ihre dummheit iibertroffen. Allerdings hat sie in Richard einen hervorragenden gegner vor sich, zugleich jedoch auch einen, dessen untaten ihr nicht krasser bekannt sein können. Aber auch wohl ein weniger überlegener mann hätte genügt, sie zu ködern. Ein mann ist jeder verstellung fähig, sobald es die eroberung eines weibes gilt. Richard hat sich's in den kopf gesetzt, sie als stufe zu seiner thronbesteigung $\mathrm{zu}$ benutzen und lä $\mathrm{sst}$ alle minen springen. $\mathrm{Er}$ lä Sst sie sich erst austoben und "setzt den stärkeren hebel erst an, als der affekt bei Anna einer gewissen erschlaffung platz gemacht hat. Er wiederholt ihr auf die verschiedenste weise, daIs er sie unendlich liebt, und sagt ihr die plumpsten schmeicheleien" 2) (bis v. 132). Dann lenkt er ihre gedanken in die richtung, in der er sie haben will, auf einen neuen gatten. Richard mufs sie wohl genug kennen, da er jahrelang am hofe gelebt hat, um zu wissen, dals und wie er sie fangen wird: "The readiest way to make the wench amends Is to become her husband and her father." ${ }^{3}$ ) Dieselbe einschätzung als einer niedrigen, feilen natur liegt in seinem schlufsmonolog. ${ }^{4}$ )

Dann wendet er sich an ihr mitleid. Wäre Anna selbst echter, so würde sie die unechtheit seiner vorgetragenen gefühle leicht merken, so aber durchschaut sie die dämonische schadenfreude, den hohn nicht, der unter seinen worten lauert. Ich kann Heine nicht beipflichten, dals Richard bei Anna zum ziel gelangt "durch all dieses", nämlich "durch schmeichelei, oder indem er ihr schrecken einflölst, oder ihr mitleid anregt, oder ihr gelegenheit gibt, sich aufzuopfern. Letzteres, nämlich das geopfertsein, ist die lieblingsrolle der weiber und kleidet sie so schön vor den leuten und gewährt ihnen in der einsamkeit so viel tränenreiche wehmutsgenüsse." ${ }^{5}$ ) Das letzte ist an sich richtig, pafst aber nicht hierher. Anna liegt

1) Vischer, a. a. 0.

3) I, 1, $127 \mathrm{f}$.

2) Wetz, a. a. 0.

4) $1,2,222-257$.

s) H. Heine, Sh.'s Mädchen und Franen: Iaady Anna. 
nichts am geopfertsein. Aber frauen sind leicht geneigt, in einem bösewicht, in den sie verliebt sind, einen unglücklichen zu wittern, einen vom schicksal verfolgten, und mitleid macht unzurechnungsfähig. Auch sehen sie sich gern in der rolle der erlöserin, ganz gleich, ob der mann überhaupt "erlöst" werden will, was meist garnicht der fall ist. Hinzu kommt, daIs frauen so empfänglich sind für den eindruck ungewöhnlicher taten, seien sie gut oder böse, die aus leidenschaft begangen sind, wie er ihr ja versichert: "But't was thy heavenly face that set me on." Auch tritt das gesetz des widerspruchs in wirkung, "was man mit aller kraft erstrebte, wird verhalst, wenn es durch zufall oder durch güte geboten wird".1) Mag sein, dafs auch ein weibischer ehrgeiz nach der krone mitspielt, dafs ihre eitelkeit eine gröIsere rolle verlangt. ${ }^{2}$ ) An ihre eitelkeit als weib wendet sich Richard auch hier nach seiner rührend vorgebrachten erzählung von seinen krokodilstränen; durch andeutung ihrer sinnlichen reize beginnt er die betörung (v. 166 ff.); denn vor allem ist es die starke sinnliche potenz ron Richards männlichkeit, die unterstiitzt durch seine äufsere erscheinung, so unglaublich es auch im ersten augenblick klingen mag - auf seinem überragenden intellekt beruht, durch die Anna sich betören lälst. Auch seine bekannte bosheit wirkt auf sie nur als männlichkeit. Trotz ihres verächtlichen blickes können wir Anna leider nicht mehr glauben, dals sie aus blofsem mitleid, wegen der gerühmten "herzensgüte", das schwert fallen läIst; denn einen augenblick später kommt das geständnis ihres wankelmuts: "I would I knew thy heart." Damit hat Richard gewonnenes spiel. Seine absolute überlegenheit, seine kühne stirn, die befriedigung ihrer eitelkeit, die angefachte sinnlichkeit paralysieren ihr schon sonst beschränktes hirn mit fülser lähmung, um so süfser, als ihr instinkt sie vor einer gefahr warnt, ihr zweifel ist eine letzte warnung. "Sie scheint schon etwas von einer bezauberung zu spüren, wie ein vogel, den der blick (!) der klapperschlange bannt." ${ }^{3}$ ) Von liebe ist auf beiden seiten keine rede, aber Anna ist begehrlich, neugierig auf neue liebe, und es hätte eines starken charakters und eines sehr klaren kopfes bedurft, hier, wenn einmal die sinn-
1) Wetr, a. a. 0.
2) Vischer, a. a. o.
3) Vischer, a. a. 0. 
lichkeit erregt war, zu widerstehen. Beides besitzt Anna nicht. Ihre gewandte rede spicht nicht gegen ihre grolse beschränktheit; hier, wie an vielen stellen bei Shakespeare, besonders in den erstlingswerken, dient die redeweise durchaus nicht zur charakterisierung der sprechenden person. Auch das gefühl der ohnmacht ist für diese willensschwache frau nur eine wollust mehr. Wie süfs ist es für charakterlose frauen, betört hinzusinken. Eine entschuldigung findet sich dafür immer.

Für Richard ist seine kunstvolle rede nur ein schachspiel, innerlich lacht er über die dumme gans, die es ihm so leicht macht. Wie fein ist der letzte schachzug, dafs er sie bittet, ihn das begräbnis wëiter besorgen zu lassen, "For divers unknown reasons". Damit fesselt er anch ihre phantasie. Grolse worte tun wunder, besonders, wenn sie nach geheimnis schmecken, frauen fallen darauf herein, weil sie eine feine witterung für grölse haben, auch für verbrecherische, wegen ihrer erlösungsmanie, und sich durch einen zusammenhang zwischen dem geheimnis und sich selber schmeicheln. Richard verstärkt durch diesen zug seine gefährliche anziehungskraft, und ihr schicksal ist besiegelt. Aber ihr ist nicht wohl dabei, nicht nur wegen ihres warnenden instinkts: wenn sie denn schon zu leicht gewonnen ist, so mufs dem wenigstens ein mäntelchen umgehängt, das dekornm gewahrt werden: sie hat ihn erhört, um dem himmel einen reuigen sünder zuzuführen: "And much it joys me, too, To see you are become so penitent" (v. 215).

Wetz entschuldigt Anna damit, dafs für sie, wie bei häufigen beispielen des mittelalters, nur das familiengefühl zu besiegen, die liebe $\mathrm{zu}$ vater und gemahl nicht so vergeistigt gewesen sei, dals sie also keine solche ungeheuerlichkeit begangen habe, indem sie Richard erhörte. ${ }^{1)}$ Aber sie ist nicht $\mathrm{zu}$ entschuldigen. Man kann wenigstens keine edle seele aus ihr machen wollen. Sie wartete darauf, versucht $\mathrm{zu}$ werden und zu fallen, darum fällt sie so leicht, wie eine schlecht verteidigte festung.

Vielleicht ist Richards dämonischer zauber ursprünglich von Sh. unbeabsichtigt in diese szene gekommen, von der alten

1) A. a. 0 . 
moralität her, als das urböse in all seiner lockung und macht. Es liegt etwas uraltes in dieser szene, das grausame spiel von katze und maus, derselbe gehalt wie in den alten tiermärchen und -fabeln, von der dummen gans, die sich vom fuchs betören läfst, vom tölpel, der vom teufel geprellt und geholt wird. Wer empfände da mitleid? Es ist ja nur eine gans. Auch Annas schicksal erweckt bei uns kein mitleid, sie wollte ja betrogen sein, es bedurfte nur des lockrufs, so ging der gimpel ins garn - ebensowenig wie das schicksal von Polonius oder der gerechten kammacher, die an Züs Bünzlin scheitern. Gewils, es ist ein erbarmungsloses geschick, aber warum soll eine Anna davon kommen, wenn eine Cordelia untergehen mufs! Annas kleines schicksal erweckt kaum interesse, was an der szene so fesselt, ist Richard in seinem höllischen glanz. Er ist "ein bluthund, der auf eine schafherde losgelassen wird. Es ist ein teil der tragödie, dafs er edler ist als die schafe, die er vernichtet. Sein ist der einzige grolse intellekt im stück. Intellekt ist immer selten. Bei königen ist er sehr selten. .... Das ansehen all dieser blntigen gräuel ist weniger schrecklich, als anzusehen, wie das schaf triumphiert, was uns modernen so lieb ist ...."1)

Wohl kann Anna nichts für ihre beschränktheit, nichts für ihren charakter, also auch nicht für ihr verderben, aber die Richard-Anna-szene ist folgerichtig aufgebaut, Annas eroberung genügend motiviert. Wem ihr los nicht begründet erscheint, der rechte mit dem schicksal, das sie so unvollkommen schuf; hier, nicht in der dichterischen fügung der szene selbst, klafit der spalt, den diese arbeit auszufüllen sich bemühte.

1) J. Masefield, Shakespeare. Home University Library p. 96/97.

Güsrrow i. M.

Gertrud Goetze. 